\title{
On the Uplink Performance of Asynchronous LAS-CDMA
}

\author{
H. Wei and L. Hanzo ${ }^{1}$ \\ School of ECS, University of Southampton, SO17 1BJ, UK. \\ Tel: +44-23-8059-3125, Fax: +44-23-8059-4508 \\ Email: $1 h^{1}$ @ecs.soton.ac.uk, http://www-mobile.ecs.soton.ac.uk
}

\begin{abstract}
In this paper closed-form formulae are derived for characterizing the BER performance of Large Area Synchronous CDMA (LAS-CDMA) as a function of both the number of resolvable paths $L_{p}$ and the maximum delay difference $\tau_{\max }$, as well as the number of users $K$, when communicating over a Nakagami- $m$ fading channel. Moreover, we comparatively studies the performance of LAS-CDMA and the traditional random code based DS-CDMA.
\end{abstract}

\section{INTRODUCTION}

In Direct Sequence Code Division Multiple Access (DS-CDMA) systems, the spreading sequences characterize the associated Inter Symbol Interference (ISI) as well as the Multiple Access Interference (MAI) properties [1]. Traditional spreading sequences, such as $m$-sequences [1], Gold codes [1] and Kasami codes [1] exhibit non-zero off-peak auto-correlations and cross-correlations, which results in a high MAI in case of asynchronous uplink transmissions. Another family of orthogonal codes is constituted by Walsh codes [1] and orthogonal Gold codes, which retain their orthogonality only in case of perfect synchronization, but they also exhibit non-zero offpeak auto-correlations and cross-correlations in asynchronous scenarios. Consequently, these correlation properties limit the achievable performance in asynchronous scenarios. Hence traditional DS-CDMA cellular systems are interference limited and suffer from the so-called 'near-far' effects, unless complex interference cancellers [1] or multi-user detectors [1] are employed for combating these adverse effects. This results in costly and 'power-hungry' implementations. All these limitations are imposed by the imperfect correlation properties of the spreading sequences employed.

The attractive family of Large Area Synchronized (LAS) CDMA spreading sequences is constituted by the combination of the so-called Large Area (LA) codes $[2,3]$ and Loosely Synchronous (LS) codes $[2,3]$. The resultant LAS codes exhibit an Interference Free Window (IFW), where the off-peak aperiodic autocorrelation values as well as the aperiodic crosscorrelation values become zero, resulting in zero ISI and zero

The work reported in this paper has formed part of the Wireless Enablers Work Area of the Core 2 Research Programme of the Virtual Centre of Excellence in Mobile \& Personal Communications, Mobile VCE, www.mobilevce.com, whose funding support, including that of EPSRC, is gratefully acknowledged. Fully detailed technical reports on this research are available to Industrial Members of Mobile VCE
MAI, provided that the time-offset of the codes is within the IFW. In this treatise we will investigate the attainable performance of LAS-CDMA in a quasi-synchronous uplink scenario, when communicating over a Nakagami- $m$ channel and characterize its BER performance as a function of both the number of resolvable paths $L_{p}$ and the maximum propagation delay $\tau_{\max }$ as well as the number of users $K$ and the Nakagami fading parameter $m$. Furthermore, we will comparatively study LAS-CDMA and traditional DS-CDMA systems.

This paper is organized as follows. Section 2 will introduce the family of LAS codes, while Section 3 will describe the LASCDMA system model. In Section 4 we will characterize the BER performance of LAS-CDMA and in Section 5 we will discuss our findings. Finally, in Section 6 we will offer our conclusions.

\section{GENERATION OF LAS-CODES}

\subsection{LA Codes}

LA codes $[2,3]$ belong to a family of ternary codes having elements of \pm 1 or 0 . Their maximum correlation magnitude is unity and they also exhibit an IFW. Let us denote the family of the $K$ number of orthogonal ternary codes employing $K$ number of binary \pm 1 pulses by $\mathrm{LA}\left(L_{A}, M, K\right)$, which exhibit a minimum spacing of $M$ chip durations between nonzero pulses, while having a total code length of $L_{A}$ chips, as shown in Figure 1. All the codes corresponding to an LA code family share the same legitimate pulse positions. However, a specific drawback of this family of sequences is their relatively low duty ratio, quantifying the density of the non-zero pulses, since this limits the legitimate variety as well as the resultant number of codes available, which inevitably limits the number of users supported. Li [2] characterized the legitimate pulse positions of the LA code family, while Choi and Hanzo [3] further increased the achievable maximum number of LA codes. In the LAS-CDMA 2000 system [4], the LA codes used constitute a modified version of the $\mathrm{LA}\left(L_{A}, M, K\right)=\mathrm{LA}(2552,136$, $17)$ code, where there are $K=17$ non-zero pulse positions, $p_{k}$, $k=0, \cdots, 16$,

For a specific procedure concerning the design of LA-code based LAS codes, please refer to $[2,3]$, where the associated correlation properties and the IFW width of the codes were also characterized.

\subsection{Loosely Synchronized Codes}

Apart from the LA codes of Section 2.1, there exists another specific family of spreading codes, which also exhibits an IFW. 


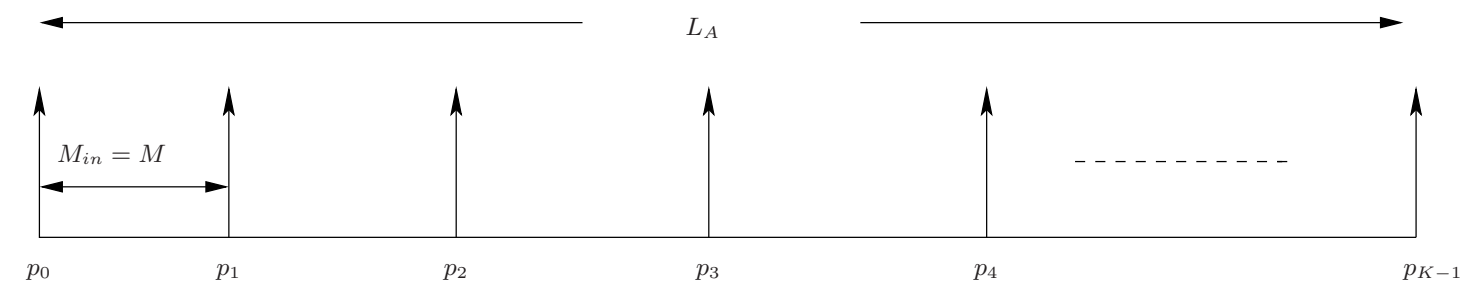

Figure 1: Stylized pulse-positions in the $\mathrm{LA}\left(L_{A}, M, K\right)$ code having $K$ number of binary \pm 1 pulses, and exhibiting a minimum spacing of $M$ chip durations between non-zero pulses, while having a total code length of $L_{A}$ chips.

Specifically, Loosely Synchronized (LS) codes [3] exploit the properties of the so-called orthogonal complementary sets [3]. To expound further, let us introduce the notation of $\operatorname{LS}\left(N, P, W_{0}\right)$ for denoting the family of LS codes, which are generated by applying a $(P \times P)$-dimensional Walsh-Hadamard $(\mathrm{WH})$ matrix to an orthogonal complementary code set of length $N$, and by inserting $W_{0}$ number of zeros both in the center and at the beginning of the complementary pair. Owing to space limitations the interested readers are referred to $[3,5]$ for further details on the family of LS codes.

\subsection{Seeding LS Codes in LA Codes to Generate LAS codes}

We observed in Section 2.1 that the main problems associated with applying LA codes in practical CDMA systems are related to their low duty ratio and to the resultant limited number of available codes. A specific family of LAS codes mitigates this problem by combining the LA codes of Section 2.1 and the LS codes of Section 2.2. More specifically, LS codes are inserted between the non-zero pulses of the LA code sequence of Figure 1, in an effort to generate an increased variety and number of spreading codes having an increased duty ratio, while maintaining attractive correlation properties. For example, in the LAS-2000 system [4] the LS spreading codes are inserted into the LA code's zero space, as shown in Figure 2 .

Let us denote the combined code generated by inserting the $\mathrm{LS}\left(N, P, W_{0}\right)$ code into the $\mathrm{LA}\left(L_{A}, M, K\right)$ code as $\operatorname{LAS}\left(L_{A}, M, K\right.$ $\left.N, P, W_{0}\right)$, which is created by employing the so-called absolute encoding method of $[2,3]$. For the sake of preserving the original IFW size of the constituent $\operatorname{LS}\left(N, P, W_{0}\right)$ code when combined with an $\mathrm{LA}\left(L_{A}, M, K\right)$ code employing the absolute encoding scheme, the length of the LS code - including $W_{0}$ number of trailing zeros - should not exceed the minimum pulse spacing $M$ of the LA code for the sake of avoiding their overlap after they were inserted into the LA code, requiring that we have:

$$
P N+2 W_{0} \leq M
$$

\section{LAS-CDMA SYSTEM MODEL}

We support $K$ asynchronous CDMA users in the system and each user is assigned an unique signature waveform $\mathbf{c}_{k}(t)=$ $\sum_{i=0}^{G-1} c_{k i} \psi_{T_{c}}\left(t-i T_{c}\right)$, where $G$ is the spreading gain and $\psi_{T_{c}}(t)$ is the rectangular chip waveform, which is defined over the interval $\left[0, T_{c}\right)$. Consequently, when the $K$ users' signals are transmitted over the frequency-selective fading channel considered, the complex low-pass equivalent signal received at a given base station can be expressed as:

$$
\begin{array}{r}
R(t)=\sum_{k=1}^{K} \sum_{l=0}^{L_{p}-1} \sqrt{2 P_{k}} \mathbf{c}_{k}\left(t-l T_{c}-\tau_{k}\right) b_{k}\left(t-l T_{c}-\tau_{k}\right) \\
\times h_{k l} \exp \left(j \theta_{k l}\right)+N(t),
\end{array}
$$

where $N(t)$ is the complex-valued low-pass-equivalent AWGN having a double-sided spectral density of $N_{0}$ and $\tau_{k}$ is the propagation delay of user $k$, while $\tau_{k}$ is assumed to be a random variable uniformly distributed in the range of $\left[0, \tau_{\max }\right]$, and $L_{p}$ is the total number of resolvable paths. $h_{k l}$ will obeys the so-called Nakagami- $m$ fading $[6,7]$.

\section{BER ANALYSIS}

Let us assume that we have achieved perfect time synchronization and that perfect estimates of the channel tap weights as well as phases are available, and coherently combine the $L$ number of path signals with the aid of the RAKE combiner, the output $Z_{k l}$ of the RAKE receiver's $l$ th finger sampled at $t=T+l T_{c}+\tau_{k}$, can be expressed as:

$$
Z_{k l}=D_{k l}+I_{k l}
$$

where $D_{k l}$ represents the desired direct component, which may be expressed as:

$$
D_{k l}=\sqrt{2 P} T_{s} b_{k}[0] h_{k l}^{2}
$$

In Equation $4 b_{k}[0]$ is the first bit transmitted by the $k$ th user, where we have $b_{k}[0] \in\{+1,-1\}$. Hence, the interference plus noise term $I_{k l}$ in Equation 3 can be expressed as:

$$
I_{k l}=I_{k l}[S]+I_{k l}[M]+N_{k},
$$

where $I_{k l}[S]$ represents the multipath interference imposed by the user-of-interest, which may be expressed as:

$$
\begin{aligned}
I_{k l}[S]= & \sqrt{2 P} T_{s} h_{k l} \sum_{\substack{l_{p}=0 \\
l_{p} \neq l}}^{L_{p}-1} \frac{h_{k l_{p}} \cos \theta_{k l_{p}}}{T_{s}} \times \\
& \int_{0}^{T_{s}} b_{k}\left[t-\left(l_{p}-l\right) T_{c}\right] \cdot c\left[t-\left(l_{p}-l\right) T_{c}\right] c[t] d t .
\end{aligned}
$$




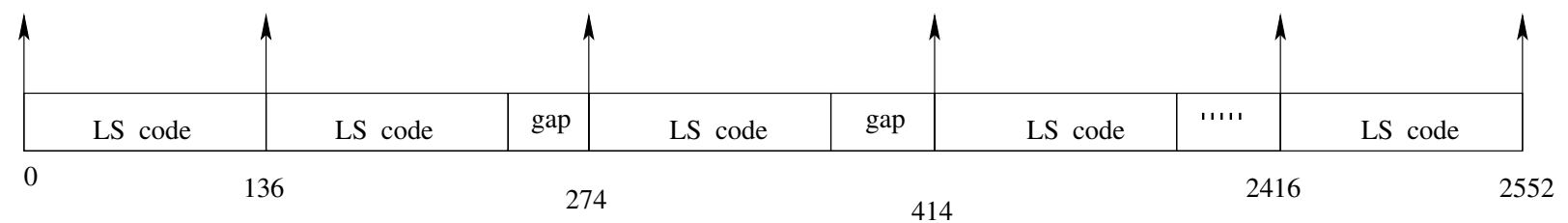

Figure 2: $\operatorname{LAS}\left(L_{A}, M, K ; N, P, W_{0}\right)$ spreading, inserting the LS codes into the zero-space of the LA codes seen Figure 1.

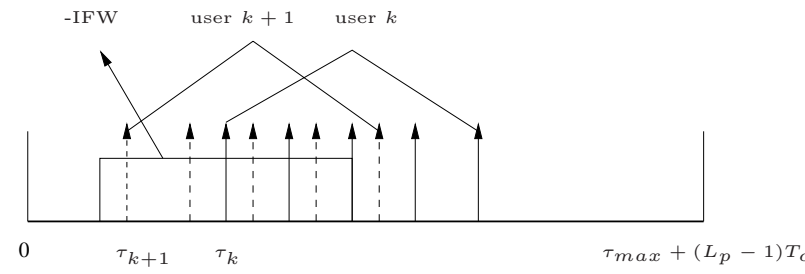

Figure 3: Illustration of the interference suppression capability of the LAS codes for the first finger of the RAKE receiver, when the width of the IFW is $\iota=2$.

Furthermore, $I_{k l}[M]$ represents the multiuser interference inflicted by the $K-1$ number of interfering signals, which may be formulated as:

$$
\begin{aligned}
I_{k l}[M]= & \sqrt{2 P} T_{s} h_{k l} \sum_{\substack{k^{\prime}=1 \\
k^{\prime} \neq k}}^{K} \sum_{l_{p}=0}^{L_{p}-1} \frac{h_{k^{\prime} l_{p}} \cos \theta_{k^{\prime} l_{p}}}{T_{s}} \\
& \cdot \int_{0}^{T_{s}} b_{k^{\prime}}\left[t-\left(l_{p}-l\right) T_{c}-\left(\tau_{k^{\prime}}-\tau_{k}\right)\right] \times \\
& c_{k^{\prime}}\left[t-\left(l_{p}-l\right) T_{c}-\left(\tau_{k^{\prime}}-\tau_{k}\right)\right] c[t] d t .
\end{aligned}
$$

In Equation 6 and Equation 7 the $\cos (\cdot)$ terms are contributed by the phase differences between the incoming carrier and the locally generated carrier used in the demodulation. Finally, the noise term in Equation 5 can be expressed as:

$$
N_{k l}=h_{k l} \int_{0}^{T_{s}} n(t) c[t] \cos \left(2 \pi f_{c} t+\theta_{k l}\right) d t,
$$

which is a Gaussian random variable having a zero mean and a variance of $N_{0} T_{s} h_{k l}^{2}$, where $\left\{h_{k l}\right\}$ represents the path attenuations.

The MRC's decision variable $Z_{k}$, which is given by the sum of all the RAKE fingers' outputs, can be expressed as:

$$
Z_{k}=\sum_{l=0}^{L_{r}-1} Z_{k l}
$$

Let us now consider the interference suppression achieved with the aid of the IFW of LAS codes. In this scenario, only the paths arriving from the interfering users outside the IFW will inflict MAI upon the reference user, and this user's own delayed paths arriving outside the IFW will additionally impose MPI. Observe in Figure 3, for example that both the $k$ th user and the $(k+1)$ st user encounter five multipath components. When we considered the $k$ th user's first RAKE receiver finger and an IFW of $\iota=2$, only those two paths of the $k t h$ user will impose MPI, which fall outside the code's IFW. Similarly, the $(k+1) s t$ user imposed only one interfering path on the $k$ th user's first RAKE finger's decision variable, since four of the five path fall within the IFW. Hence, we will investigate the interference suppression capability of the IFW, as shown in a stylized fashion in Figure 3.

Given a fading attenuation set of $\left\{h_{k l}, l=0,1, \ldots, L_{r}-1\right\}$, it can be shown that the BER of the LAS-CDMA system may be expressed as:

$$
P_{b}(\gamma)=Q\left(\sqrt{\sum_{l=0}^{L_{r}-1} 2 \gamma_{l}}\right)
$$

where $Q(x)$ represents the Gaussian $Q$-function, which can also be formulated as [7] $Q(x)=\frac{1}{\pi} \int_{0}^{\pi / 2} \exp \left(-\frac{x^{2}}{2 \sin ^{2} \theta}\right) d \theta$, where we have $x \geq 0$. Furthermore, $2 \gamma_{l}$ in Equation 10 represents the output Signal to Interference plus Noise Ratio (SINR) at the $l$ th finger of the RAKE receiver, while $\gamma_{l}$ is given by:

$$
\gamma_{l}=\gamma_{c} \cdot \frac{h_{k l}^{2}}{\Omega_{0}} .
$$

The expression of $\gamma_{c}$ may be formulated as:

$$
\gamma_{c}=\left[\frac{\Upsilon_{S}(l)}{G}+\frac{2 K \Upsilon_{M}(l)}{3 G}+\left(\frac{\Omega E_{b}}{N_{0}}\right)^{-1}\right]^{-1} .
$$

The MPI and MAI interference reduction factors of $\Upsilon_{S}(l)$ and $\Upsilon_{M}(l)$ are given as

$$
\begin{gathered}
\Upsilon_{S}(l)=2 G \cdot \sum_{\substack{l_{p}=0 \\
\left|l_{p}-l\right|>\iota}}^{L_{p}-1} e^{-\eta l_{p}}\left[\rho_{k k}^{2}(\xi)+\varrho_{k k}^{2}(\xi)\right], \\
\Upsilon_{M}(l)=\frac{3 G}{K} \sum_{\substack{k^{\prime}=1 \\
k^{\prime} \neq k}}^{K} \int_{0}^{\tau_{\max }} \int_{0}^{\tau_{\max }} \frac{1}{\tau_{\max }} \frac{1}{\tau_{\max }} \times \\
\sum_{\substack{l_{p}=0 \\
L_{p}-1}}^{L^{-\eta l_{p}}\left(\hat{\rho}_{k^{\prime} k}^{2}\left(\tau_{c}\right)+\hat{\varrho}_{k^{\prime} k}^{2}\left(\tau_{c}\right)\right) d \tau_{k} d \tau_{k^{\prime}},}
\end{gathered}
$$

where the $\rho_{k k}^{2}(\cdot)$ and $\varrho_{k k}^{2}(\cdot)$ are the partial auto-correlations of the LAS-codes [8], while $\hat{\rho}_{k^{\prime} k}\left(\tau_{c}\right)$ and $\hat{\rho}_{k^{\prime} k}\left(\tau_{c}\right)$ are the partial cross-correlations of the LAS-codes [8].

For the random code based DS-CDMA system [9], the corresponding $\gamma_{c}$ expression is given by [9]:

$$
\gamma_{c}=\left[\frac{q\left(L_{p}, \eta\right)-1}{G}+\frac{2 K q\left(L_{p}, \eta\right)}{3 G}+\left(\frac{\Omega E_{b}}{N_{0}}\right)^{-1}\right]^{-1},
$$

where we have $q\left(L_{p}, \eta\right)=\sum_{l=0}^{L_{p}-1} e^{-\eta l}$. 


\subsection{Bit Error Probability Analysis}

The average BER, $P_{b}(E)$ can be obtained by the weighted averaging of the conditional BER expression of Equation 10 over the joint $\mathrm{PDF}$ of the instantaneous $\mathrm{SNR}$ values corresponding to the $L_{r}$ multipath components $\left\{\gamma_{l}: l=1,2, \ldots, L_{r}\right\}$. Since the random variables $\gamma_{l}$ are assumed to be statistically independent, the average BER expression of Equation 10 may be formulated as [7]:

$$
P_{b}(E)=\frac{1}{\pi} \int_{0}^{\pi / 2} \prod_{l=0}^{L_{r}-1} I_{l}\left(\bar{\gamma}_{l}, \theta\right) d \theta,
$$

where we have

$$
I_{l}\left(\bar{\gamma}_{l}, \theta\right)=\int_{0}^{\infty} \exp \left(-\frac{\gamma_{l}}{\sin ^{2} \theta}\right) p_{\gamma_{l}}\left(\gamma_{l}\right) d \gamma_{l} .
$$

Since we have $\gamma_{l}=\gamma_{c} \cdot \frac{\left(h_{l}\right)^{2}}{\Omega_{0}}$ and $h_{l}$ obeys the Nakagami$m$ distribution, it can be shown that the PDF of $\gamma_{l}$ can be formulated as:

$$
p_{\gamma_{l}}\left(\gamma_{l}\right)=\left(\frac{m_{l}}{\bar{\gamma}_{l}}\right)^{m_{l}} \frac{\gamma^{m_{l}-1}}{\Gamma\left(m_{l}\right)} \exp \left(-\frac{m_{l} \gamma_{l}}{\bar{\gamma}_{l}}\right), \quad \gamma_{l} \geq 0,
$$

where $\bar{\gamma}_{l}=\gamma_{c} e^{-\eta l}$ for $l=0,1, \ldots, L_{r}-1$.

Upon substituting Equation (18) into Equation (17) it can be shown that we have [7]:

$$
I_{l}\left(\bar{\gamma}_{l}, \theta\right)=\left(\frac{m_{l} \sin ^{2} \theta}{\bar{\gamma}_{l}+m_{l} \sin ^{2} \theta}\right)^{m_{l}}
$$

Finally, upon substituting Equation (19) into Equation (16), the average BER of both the random and LAS-code based CDMA system can be written as:

$$
P_{b}(E)=\frac{1}{\pi} \int_{0}^{\pi / 2} \prod_{l=0}^{L_{r}-1}\left(\frac{m_{l} \sin ^{2} \theta}{\bar{\gamma}_{l}+m_{l} \sin ^{2} \theta}\right)^{m_{l}} d \theta .
$$

\section{PERFORMANCE OF LAS DS-CDMA}

In our investigations we compared a traditional and a LAScode based CDMA system, both of which have the same chiprate and bandwidth. In the LAS-CDMA 2000 system, the $\mathrm{LA}(2552,136,17)$ and $\operatorname{LS}(4,32,4)$ codes are combined, as seen in Figure2. Moreexplicitly, thetotallengthoftheLS $\left(N, P, W_{0}\right)=\mathrm{LS}(4,32,4)$ code is $L_{s}=N P+2 W_{0}=136$ chips, which is incorporated into the $\mathrm{LA}\left(L_{A}, M, K\right)=\mathrm{LA}(2552,136,17)$ code. Although the length of this LS code is $L_{S}=N P+2 W_{0}=136$, the effective spreading gain of the LAS code is identical to that of its constituent LS codes, namely $G_{\mathrm{LAS}}=G_{\mathrm{LS}}=128$. By contrast, a traditional random code based CDMA system having the same $L_{A}=2552$ chips would have a higher spreading gain, since it does not have any zero-valued gaps, nor has an IFW. Hence the corresponding spreading gain becomes $G_{\text {Random }}=2552 / 17=151$. For simplicity's sake, we assume that all paths have the same Nakagami fading parameter, i.e. we have $m_{l}=m, l=0, \ldots, L_{r}-1$.

Figure 4 exhibits the performance of these two systems communicating over different fading channels associated with various Nakagami fading parameters. More explicitly, when we have $m=1$, we model a Rayleigh fading channel, $m=2$ represents a Rician fading channel, while $m \rightarrow \infty$ corresponds

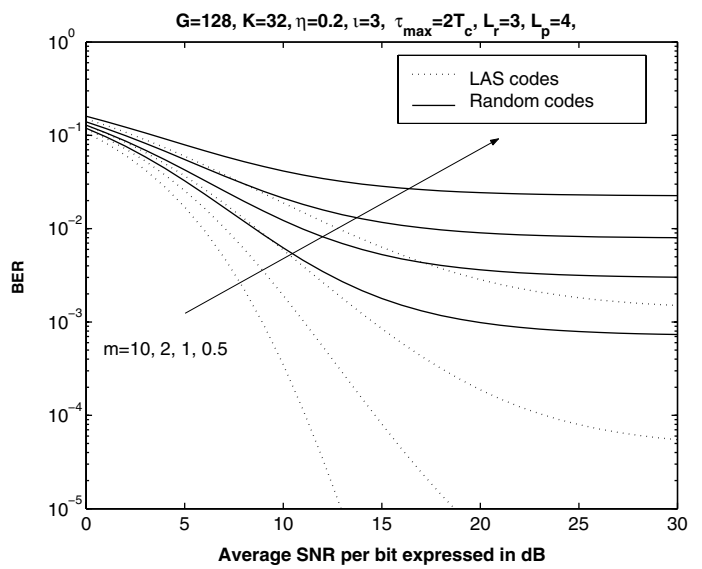

Figure 4: BER versus channel SNR performance comparison of random code based classic CDMA and LAS CDMA, when communicating over different Nakagami fading channels.

to an AWGN channel. We can observe from Figure 4 that the LAS-CDMA scheme exhibited a significantly better BER performance than the traditional DS-CDMA system, regardless of the value of $m$. More specifically, provided that all these uplink users are in a quasi-synchronous state, $i$.e we have $\tau_{\max }=2 T_{c}$ and $L_{p}=4$, the LAS-CDMA scheme outperformed the traditional DS-CDMA system, when communicating over different Nakagami multipath fading channels.

Figure 5 shows the performance of these two systems for transmission over different dispersive channels having $L_{p}=$ $4 \ldots 12$ resolvable multipath components and assuming that $L_{r}=3$ of these components were actually combined by the RAKE receiver owing to its complexity limitation. We can observe from Figure 5 that when the channel became more dispersive, the LAS-CDMA system's performance was significantly degraded and its gain over the traditional DS-CDMA system was eroded. Nonetheless, the LAS-CDMA scheme still outperformed the traditional DS-CDMA system, provided that the users were in a quasi-synchronous state, i.e when we had $\tau_{\max }=2 T_{c}$.

In Figure 6 we can observe that as the maximum propagation delay $\tau_{\max }$ increases, the performance of LAS-CDMA significantly degrades. In the strongly dispersive scenario, when we have $\tau_{\max } \geq 10 T_{c}$, the LAS-CDMA system's performance becomes inferior in comparison to that of traditional DS-CDMA. This is because the insertion of zeros in the LAS codes reduces the effective spreading gain of the LAS-CDMA system and when the delay difference $\tau_{\max }$ increases, the MAI suppression capability will be inevitably reduced. Furthermore, outside the IFW their correlation properties are worse than those of classic spreading codes.

In Figure 7 , we can observe that for $K \geq 60$ users the LAS-CDMA system will have no advantage in comparison to the traditional DS-CDMA scheme, or may even perform worse than the traditional DS-CDMA arrangement, although all users operate in a quasi-synchronous manner. Hence, we may conclude that the employment of LAS-CDMA is beneficial in low user load scenarios, where the delay spread is also limited. 


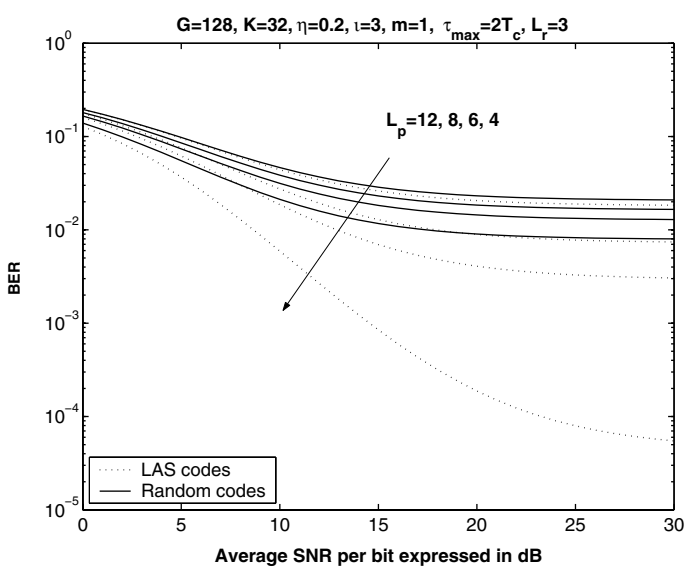

Figure 5: BER versus channel SNR performance comparison of random code based classic CDMA and LAS CDMA over different dispersion channels.

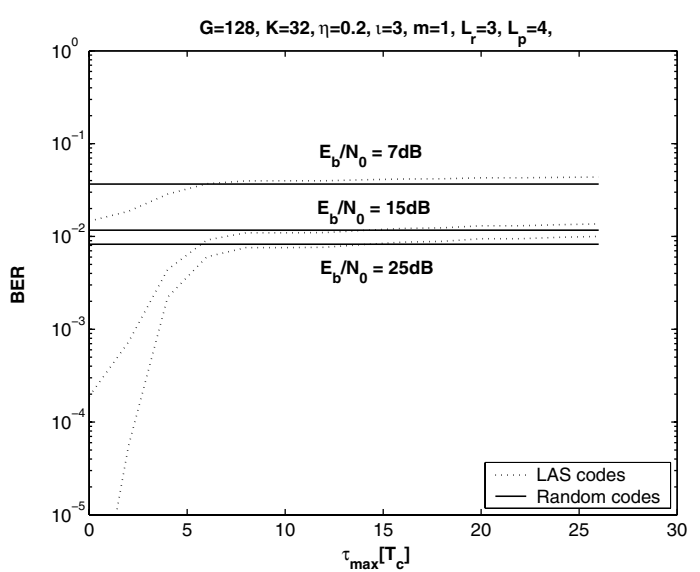

Figure 6: BER performance comparison of random code based classic CDMA and LAS CDMA as a function of the maximum delay difference $\tau_{\max }$.

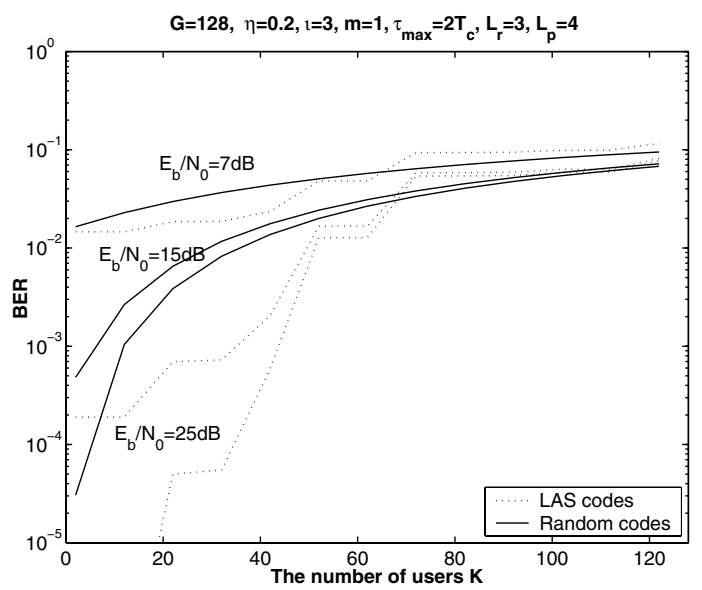

Figure 7: BER performance comparison of random code based classic CDMA and LAS CDMA as a function of the number of users $K$ supported.

\section{CONCLUSION}

In conclusion, LAS-CDMA was investigated, which exhibited a significantly better performance than the traditional random code based DS-CDMA system in a relatively low chip-rate scenario, provided that all users operate in a quasi-synchronous manner. As the chip-rate increases, the number of resolvable paths also increases, which will impose a performance degradation. Hence, as suggested in Figure 5, LAS-CDMA may be expected to have a moderate performance gain over the traditional DS-CDMA system, when $L_{p}$ is in excess of 12 . Furthermore, the limited number of available LAS codes having a certain IFW width suggests that the employment of LASCDMA is beneficial in a low-user-load scenario.

\section{REFERENCES}

[1] L. Hanzo, L. L. Yang, E. L. Kuan, and K. Yen, Single- and Multi-Carrier DS-CDMA. John Wiley and IEEE Press, 2003, 1060 pages.

[2] D. Li, "A High Spectrum Efficient Multiple Access Code," Chinese Journal of Electronics, vol. 8, pp. 221-226, Jul. 1999.

[3] B. J. Choi and L. Hanzo, "On the Design of LAS Spreading Codes," in IEEE VTC 2002 Fall Conference, (Vancouver, Canada), pp. 2172-2176, Sept. 2002.

[4] CWTS/China, Physical Layer Specification for LAS-2000, June 2000.

[5] S. Stańczak, H. Boche, and M. Haardt, "Are LAS-codes a Miracle?," in GLOBECOM '01, vol. 1, (San Antonio, Texas), pp. 589-593, Nov. 2001.

[6] J. G. Proakis, Digital Communications. Mc-Graw Hill International Editions, 3rd ed., 1995.

[7] M.-S. Alouini and A. J. Goldsmith, "A Unified Approach for Calculating Error Rates of Linearly Modulated Signals over Generalized Fading Channels," IEEE Transactions on Communications, vol. 47, pp. 1324-1334, September 1999.

[8] E. Geraniotis and B. Ghaffari, "Performance of Binary and Quaternary Direct-Sequence Spreading-Spectrum Multiple-Access Systems with Random Signature Sequences," IEEE Transactions on Communications, vol. 39, pp. 713-724, May 1991.

[9] T. Eng and L. B. Milstein, "Coherent DS-CDMA Performance in Nakagami Multipath Fading," IEEE Transactions on Communications, vol. 43, pp. 1134-1143, Feb./Mar./Apr. 1995. 